\title{
Supplementary data: External validation of the ProCaRS nomograms and comparison of existing risk-stratification tools for localized prostate cancer
}

David Tiberi, $M D^{1}$; George Rodrigues, $M D^{2}$; Tom Pickles, $M D^{3}$; Jim Morris, $M D^{3}$; Juanita Crook, $M D^{4}$;

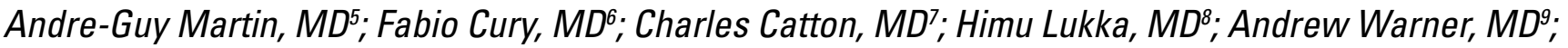
Daniel Taussky, $M D^{\prime}$

'Department of Radiation Oncology, Centre Hospitalier de I'Université de Montréal (CHUM), Hôpital Notre-Dame, Montreal, QC; ${ }^{2}$ Department of Oncology, London Health Sciences Centre; Schulich School of Medicine \& Dentistry, Western University, London, ON; ${ }^{2}$ Department of Radiation Oncology, BC Cancer Agency, Vancouver Centre, University of British Columbia, Vancouver, BC; ${ }^{2}$ Department of Radiation Oncology, BC Cancer Agency, Kelowna, BC; ${ }^{5}$ Department of Radiation Oncology, Centre Hospitalier Universitaire de Québec-L'Hôtel-Dieu de Québec, Quebec City, QC; ${ }^{6}$ Department of Oncology, Division of Radiation Oncology, McGill University Health Centre, Montreal, QC; ${ }^{7}$ Radiation Medicine Program, Princess Margaret Cancer Centre, Toronto, ON; ${ }^{8}$ Department of Radiation Oncology, Juravinski Cancer Centre, Hamilton, ON; ${ }^{D}$ Department of Radiation Oncology, London Health Sciences Centre, London, ON; Canada Published online April 11, 2017

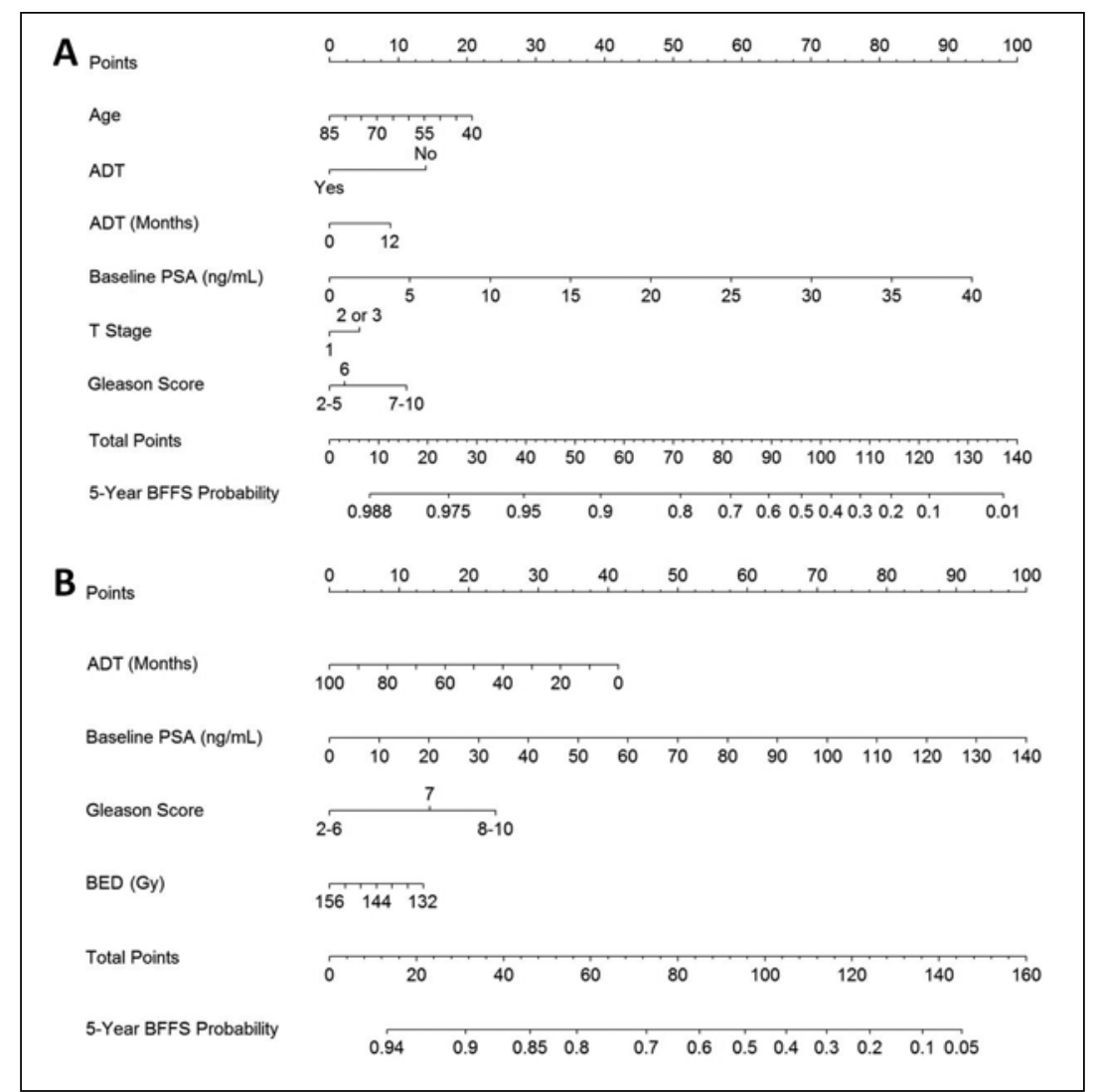

Supplementary Fig. 1. ProCaRS nomograms predicting five-year ASTRO II Phoenix biochemical failure-free survival for (A) LDR-brachytherapy only ( $n=4208$ ); and (B) EBRT only (reproduced with permission from Cureus). ADT: androgen-deprivation therapy; BED: biologically effective dose; BFFS: biochemical failure-free survival; PSA: prostate-specific antigen. 


\begin{tabular}{|c|c|c|c|c|c|}
\hline \multicolumn{6}{|c|}{$\begin{array}{l}\text { Supplementary Table } 1 \text {. Summary of actuarial estimates for bioch } \\
\text { risk stratification variable for the CHUM database }(n=2266)\end{array}$} \\
\hline Group & Median (Years) & $3 Y(\%)$ & $4 Y(\%)$ & $5 Y(\%)$ & $10 Y(\%)$ \\
\hline \multicolumn{6}{|c|}{ Biochemical-failure-free survival } \\
\hline All patients & NR & 97.4 & 95.2 & 92.9 & 72.0 \\
\hline \multicolumn{6}{|l|}{ CAPRA-3 } \\
\hline Low & NR & 98.7 & 97.9 & 96.6 & 82.7 \\
\hline Intermediate & NR & 96.6 & 94.7 & 91.7 & 70.0 \\
\hline High & NR & 95.3 & 87.9 & 85.2 & 62.3 \\
\hline \multicolumn{6}{|l|}{ ProCaRS, n (\%) } \\
\hline Low & NR & 98.3 & 97.3 & 96.2 & 82.1 \\
\hline Low-intermediate & NR & 97.1 & 94.8 & 91.7 & 65.5 \\
\hline High-intermediate & NR & 94.7 & 90.2 & 86.4 & 70.2 \\
\hline High & NR & 96.9 & 94.3 & 92.9 & 74.6 \\
\hline Very high & 9.34 & 95.1 & 87.1 & 82.4 & 45.8 \\
\hline \multicolumn{6}{|l|}{ GUROC, n (\%) } \\
\hline Low & NR & 98.3 & 97.3 & 96.2 & 82.1 \\
\hline Intermediate & NR & 96.8 & 94.2 & 91.0 & 68.3 \\
\hline High & NR & 96.2 & 91.5 & 88.8 & 65.2 \\
\hline \multicolumn{6}{|l|}{$\mathrm{NCCN}^{1,2}$} \\
\hline Low & NR & 98.4 & 97.5 & 96.2 & 81.8 \\
\hline Intermediate & NR & 96.7 & 94.2 & 91.1 & 68.3 \\
\hline High & NR & 96.0 & 92.7 & 90.0 & 65.2 \\
\hline \multicolumn{6}{|l|}{ Overall survival } \\
\hline All patients & NR & 97.9 & 96.2 & 94.7 & 80.6 \\
\hline \multicolumn{6}{|l|}{ CAPRA-3 } \\
\hline Low & NR & 99.0 & 98.3 & 98.1 & 94.4 \\
\hline Intermediate & NR & 97.4 & 94.8 & 93.5 & 77.8 \\
\hline High & NR & 96.4 & 94.3 & 89.2 & 72.6 \\
\hline \multicolumn{6}{|l|}{ ProCaRS, n (\%) } \\
\hline Low & NR & 99.1 & 98.4 & 97.9 & 96.5 \\
\hline Low-intermediate & NR & 97.4 & 95.0 & 93.4 & 72.1 \\
\hline High-intermediate & NR & 95.0 & 92.2 & 90.2 & 85.6 \\
\hline High & NR & 98.3 & 97.1 & 95.6 & 78.6 \\
\hline Very high & 11.84 & 96.9 & 91.3 & 85.1 & 69.5 \\
\hline \multicolumn{6}{|l|}{ GUROC, n (\%) } \\
\hline Low & NR & 99.1 & 98.4 & 97.9 & 96.5 \\
\hline Intermediate & NR & 96.9 & 94.5 & 92.9 & 75.4 \\
\hline High & NR & 97.7 & 94.7 & 91.3 & 74.8 \\
\hline \multicolumn{6}{|l|}{$\mathrm{NCCN}^{1,2}$} \\
\hline Low & NR & 99.3 & 98.7 & 98.1 & 96.7 \\
\hline Intermediate & NR & 96.8 & 94.4 & 92.8 & 75.6 \\
\hline High & NR & 97.6 & 95.3 & 91.8 & 77.2 \\
\hline
\end{tabular}

